Review Article

\title{
lncRNA-Triggered Macrophage Inflammaging Deteriorates Age-Related Diseases
}

\author{
Lulingxiao Nie $\mathbb{C}^{1,2}$ Peng Zhang, ${ }^{1,2}$ Qian Wang, ${ }^{1,2}$ Xinyi Zhou, ${ }^{1,2}$ and Qi Wang $\mathbb{D}^{1,2}$ \\ ${ }^{1}$ State Key Laboratory of Oral Diseases, National Clinical Research Center for Oral Diseases, West China Hospital of Stomatology, \\ Sichuan University, China \\ ${ }^{2}$ Department of Prosthodontics, West China Hospital of Stomatology, Sichuan University, China
}

Correspondence should be addressed to Qi Wang; wqinno8751@gmail.com

Received 16 September 2019; Revised 10 November 2019; Accepted 13 November 2019; Published 21 December 2019

Academic Editor: Helen C. Steel

Copyright (C) 2019 Lulingxiao Nie et al. This is an open access article distributed under the Creative Commons Attribution License, which permits unrestricted use, distribution, and reproduction in any medium, provided the original work is properly cited.

Aging and age-related diseases (ARDs) share basic mechanisms largely involving inflammation. A chronic, low-grade, subclinical inflammation called inflammaging occurs during aging. Autophagy defects, oxidative stresses, senescence-associated secretory phenotypes (SASPs), and DNA damage generally contribute to inflammaging and are largely regulated by numerous lncRNA through two-level vicious cycles disrupting cellular homeostasis: (1) inflammaging and the cellular senescence cascade and (2) autophagy defects, oxidative stress, and the SASP cascade. SASPs and inflammasomes simultaneously cause inflammaging. This review discusses the involvement of macrophage inflammaging in various ARDs and its regulation via lncRNA. Among macrophages, this phenomenon potentially impairs its immunosurveillance and phagocytosis mechanisms, leading to decreased recognition and clearance of malignant and senescent cells. Moreover, SASPs extracellularly manifest to induce paracrine senescence. Macrophage senescence escalates to organ level malfunction, and the organism is more prone to ARDs. By targeting genes and proteins or functioning as competing endogenous RNA (ceRNA), lncRNA regulates different phenomena including inflammaging and ARDs. The detailed mechanism warrants further elucidation to obtain pathological evidence of ARDs and potential treatment approaches.

\section{Introduction}

Rapid scientific and technological advancements have markedly increased the lifespan of humans, along with an inevitable increase in the prevalence of age-related diseases (ARDs) including cancer and diabetes mellitus, wherein age constitutes the primary risk factor and the prevalence of which increases with age [1]. Although the origin of ARDs has long been investigated, the underlying mechanisms remain unclear. Aging occurs throughout an individual's lifespan [2-4]. Numerous theories have been postulated, among which inflammaging is potentially a prominent contributor to ARDs and an appealing therapeutic target [5].

Inflammaging is defined as a chronic, systematic, lowgrade, subclinical proinflammatory environment that accelerates cellular aging [6] and occurs in various ARDs including neurodegeneration-related diseases, metabolic diseases, and atherosclerosis [6, 7]. Cellular senescence and SASPs have been suggested as the two major contributors to inflammaging [8]. Senescent cells produce interleukins, chemokines, growth factors, and proteases, which together constitute the SASP $[8,9]$. Macrophages are prototypical immune cells expressing SASPs [10]. SASPs are critical for cell vitality and changes in macrophages. Macrophage's polarization or function are associated with numerous disorders including ARDs [11-13].

lncRNAs regulate different biological phenomena. Numerous putative lncRNAs, which indeed encode micropeptides, have been reported [14], prompting studies on the complexity and importance of these previously disregarded molecules. Advancements in lncRNA-induced ARDs have been reported; however, the mechanism underlying lncRNA regulation of inflammaging remains unknown. This review discusses one potential mechanism wherein lncRNA triggers 


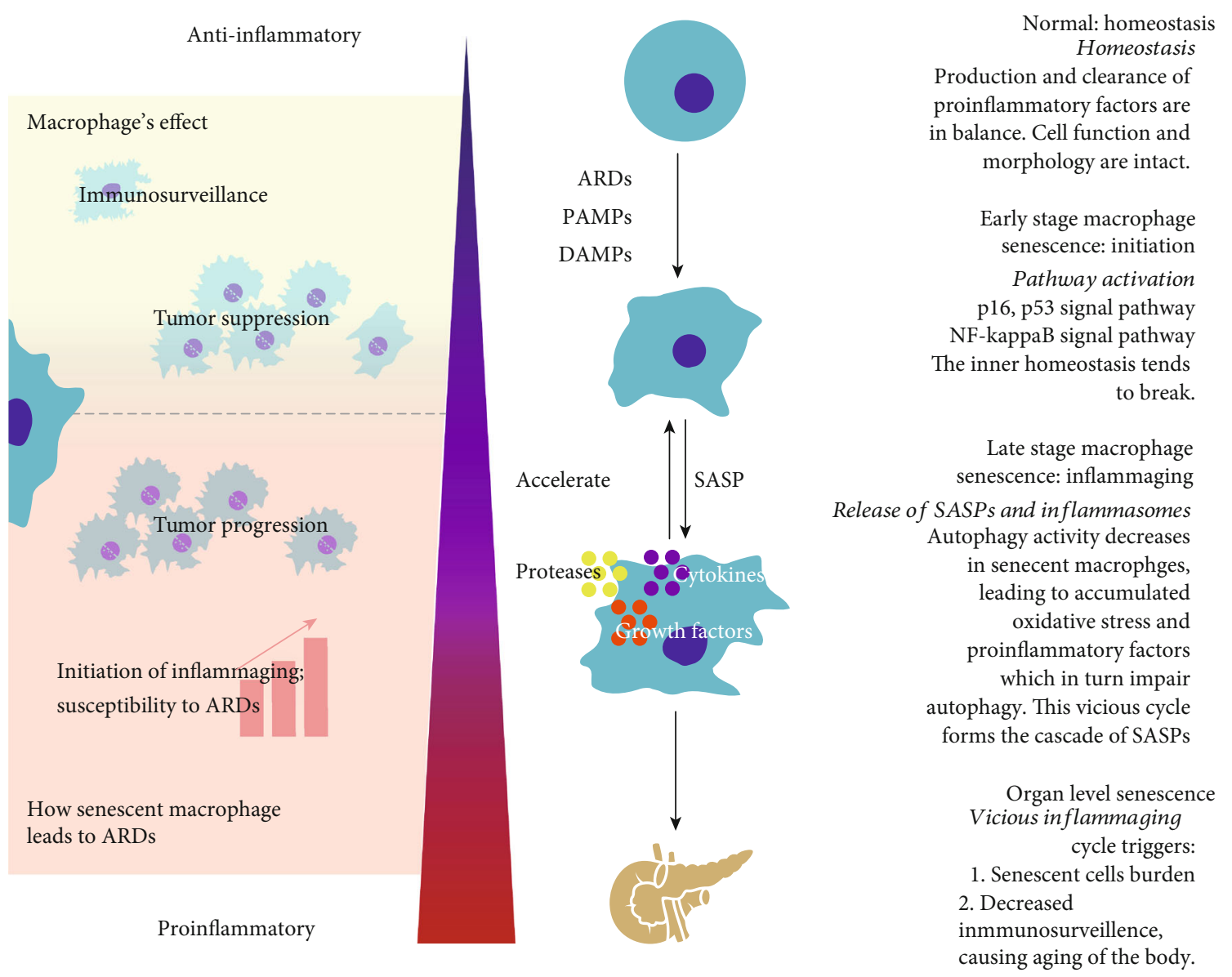

Figure 1: Schematic of the process of cellular senescence and its dual effects.

cellular senescence and the SASP to exacerbate ARDs, thus potentially providing pathological evidence of ARDs and methods for their treatment.

\section{Macrophage Senescence and Inflammaging: An Intimate Relationship}

Cellular senescence is a particularly stable state of permanent cell cycle arrest. Macrophages, although terminally differentiated cells, do not undergo this type of replicative senescence and may hence undergo stress-induced senescence. This concept was first introduced in vitro, and it is widely accepted that stress (including reactive oxygen species [ROS] and autophagy defects) plays an important role in senescence in vivo [15]. Senescence occurs throughout an individual's lifespan and plays diverse critical roles. Senescent cells undergo a characteristic alteration wherein morphological changes, functional impairment, and the expression of senescence-associated $\beta$-galactosidase (SA- $\beta$-GAL) and p $16^{\text {Ink4a }}$ could be detected [16-18]. For immunocytes including macrophages, aging impairs their capabilities and induces immunodeficiency to some extent $[19,20]$, leading to reduced immunosurveillance and phagocytosis and resulting in immune evasion among malignant tumors. However, considering the decreased capability of recognizing and clearing senescent cells, the space that normal cells should have occupied is in turn occupied by senescent cells, characterized by a reduction in overall physiological function. Consequently, the organism would be more prone to ARDs.

In healthy conditions, macrophages maintain homeostasis; however, in pathological states, different stresses including DNA damage, telomere shortening, oncogene activation [21, 22], impairment of some key proteins [23], and infections activate the p53, AIM2, and NF- $\kappa$ B signal pathways $[24,25]$, initiating macrophage senescence. Telomeric DNA is prone to various types of damage including oxidative stress, which can induce telomere shortening [26]. Hence, even terminally differentiated macrophages undergo telomere dysfunction, triggering DNA damage response (DDR) pathways and finally causing cellular senescence [27]. When these damage-associated molecule patterns (DAMPs) and pathogen-associated molecular patterns (PAMPs) are highly intensive or temporally irreversible, the balance between the production and clearance of proinflammatory factors is disrupted. At later stages of macrophage senescence, the net effect is SASP expression (Figure 1). These factors not only aggravate macrophage senescence but are also extracellularly released, thus impairing the functions of surrounding cells. This process is called "paracrine senescence" [28] and causes a wider range of inflammaging. With steady accumulation of senescent cells, senescence eventually occurs at the cellular level and then 
at the organ level, causing organ malfunction, consequently resulting in corresponding aging phenotypes.

Macrophage senescence is inextricably associated with inflammaging, bridging SASPs and inflammasomes. p16 ${ }^{\text {Ink4a }}$ has been implicated in macrophage activation or polarization [29], and SASPs induce senescence-like phenotypes in macrophages [30]. Senescence-related changes in macrophages are speculated to generally represent their proinflammatory activation [31], i.e., proinflammatory effects of macrophages are accompanied by inflammaging to some extent. SASPs induce a proinflammatory and aging-promoting environment. This constant detrimental stimulation potentially threatens various cellular components, including organelles and DNA [7]. By increasing SASP expression, these impaired components aggravate inflammaging and consequently impair inflammatory homeostasis, thus accelerating senescence and the susceptibility to ARDs [32]. SASPs and inflammasomes simultaneously cause inflammaging: they trigger inflammaging, thus accelerating aging, and inflammaging is manifested through inflammasome induction and SASP expression. Moreover, autophagy defects, oxidative stress, and DNA damage result in the assembly of inflammasomes and expression of SASPs [31-33].

\section{IncRNA Triggers Macrophage Senescence in ARDs}

Emerging data suggest that lncRNA plays a key role in regulating inflammatory responses. Alterations in various lncRNA expression levels are associated with a proinflammatory phenotype in various ARDs [34-36]. This leads to modification of cellular senescence through several diverse approaches, whether by mediating gene expression or protein function or functioning as competing endogenous RNA (ceRNA). Changes in lncRNAs in ARDs and the corresponding consequences have been widely studied, especially in cancer. However, the association between lncRNA and cellular senescence in ARDs remains an interesting and complex issue. Here, we consider macrophage senescence to investigate the mechanism underlying lncRNA-mediated induction and exacerbation of ARDs.

3.1. Diabetes Mellitus. Diabetes mellitus is among the most serious recent public health challenges. Changes in macrophage expression profiles exert local and systemic inflammatory stress. IncRNA E330013P06 regulates proximal genes involved in macrophage functions to increase IL-6, TNF- $\alpha$, and NOS2 levels while downregulating anti-inflammatory cytokines [37]. Besides gene regulation, Lethe and Dnm3os reportedly bind to the p65 subunit and induce epigenetic modifications, thus disrupting nuclear translocation of NF$\kappa \mathrm{B}$ and enhanced inflammatory responses and oxidative stress $[38,39]$. Moreover, changes in certain lncRNA expression levels potentially lead to higher, more lethal inflammation [40]. In diabetic complications, MALAT1 potentially triggers pyroptosis in macrophages, thus exacerbating the severe consequences of atherosclerosis [40]. These molecular alterations suggest the disruption of macrophage homeosta- sis with the direct outcome that pancreatic $\beta$ cells and focal lesions may undergo harsher damage.

3.2. Cancer. As shown in Table 1, macrophage-associated cancers are mostly derived from epithelia. A recent theory states that tumor growth depends not only on tumor cells themselves but also on the peripheral cellular and noncellular components [41]. In cancers, macrophages are recruited to the lesion [42]. Such tumor infiltration and the immunosurveillance of macrophages render them an important cell type in cancer. lncRNAs regulate cancer primarily through direct alteration of gene expression. LNMAT1 recruits hnRNPL to the CCL2 promoter, thus altering its expression and mediating epigenetic alterations and activating and recruiting macrophages to the site of bladder cancer, promoting tumor invasion and lymphatic metastasis [43]. Furthermore, tumor cell-derived lncRNAs are released via exosomes and are internalized by the surrounding macrophages. Changes to downstream pathways disrupt their function, especially phagocytosis, which is critical for tumor clearance [44]. As ceRNA, NIFK-AS1 and CCAT1 decoyed microRNA to suppress macrophage M2 polarization and malignant behaviors $[45,46]$. CCAT1 expression levels differ between M1 and M2 macrophages. Furthermore, lncRNA Cox2 potentially alters M1/M2 polarization, thus preventing immune evasion and metastasis [47]. These reports indicate that macrophage M2 polarization facilitates malignant behaviors and some lncRNAs are essential in maintaining the cellular phenotype.

3.3. Atherosclerosis and Related Heart Disease. Atherosclerosis is a chronic inflammatory disease. Macrophages have been recently reported to display marked inflammatory plasticity, particularly polarization. They perpetuate chronic inflammation and growth of atherosclerotic plaques, thus being central to the initiation, growth, and ultimately the rupture of arterial plaques [48]. Studies on atherosclerosis and macrophage have reported that lncRNAs majorly function as ceRNA in causing atherosclerosis. By sequestering microRNAs, MITA, GAS5, HOTAIR, and UCA1 promote M1 polarization, inducing proinflammatory cytokine, matrix metalloproteinase, and ROS levels [49-52]. Furthermore, MeXis and CDKN2B-AS1 interact with DDX17 and DNMT1 to modulate downstream gene expression, thus altering macrophage function and polarization $[53,54]$. Atherosclerosis contributes to various lesions, especially cardiovascular disease. Current evidence suggests that the effect of lncRNAs on macrophages in coronary artery disease is the same as that on atherosclerosis [55], highlighting the consistency of its function and prompting its potential as a therapeutic target.

3.4. Other ARDs. Besides the aforementioned diseases, lncRNA regulation of macrophage senescence is also reflected in other ARDs, primarily osteoarthropathies. lncRNAs generally function in the same manner in these diseases as in diabetes, cancer, and atherosclerosis. They alter the expression or function of key proteins by targeting genes or proteins or functioning as ceRNAs to disrupt homeostasis and engage in cellular senescence. Although these are indeed phenotypes of aging cells, they are indirect. A study on 


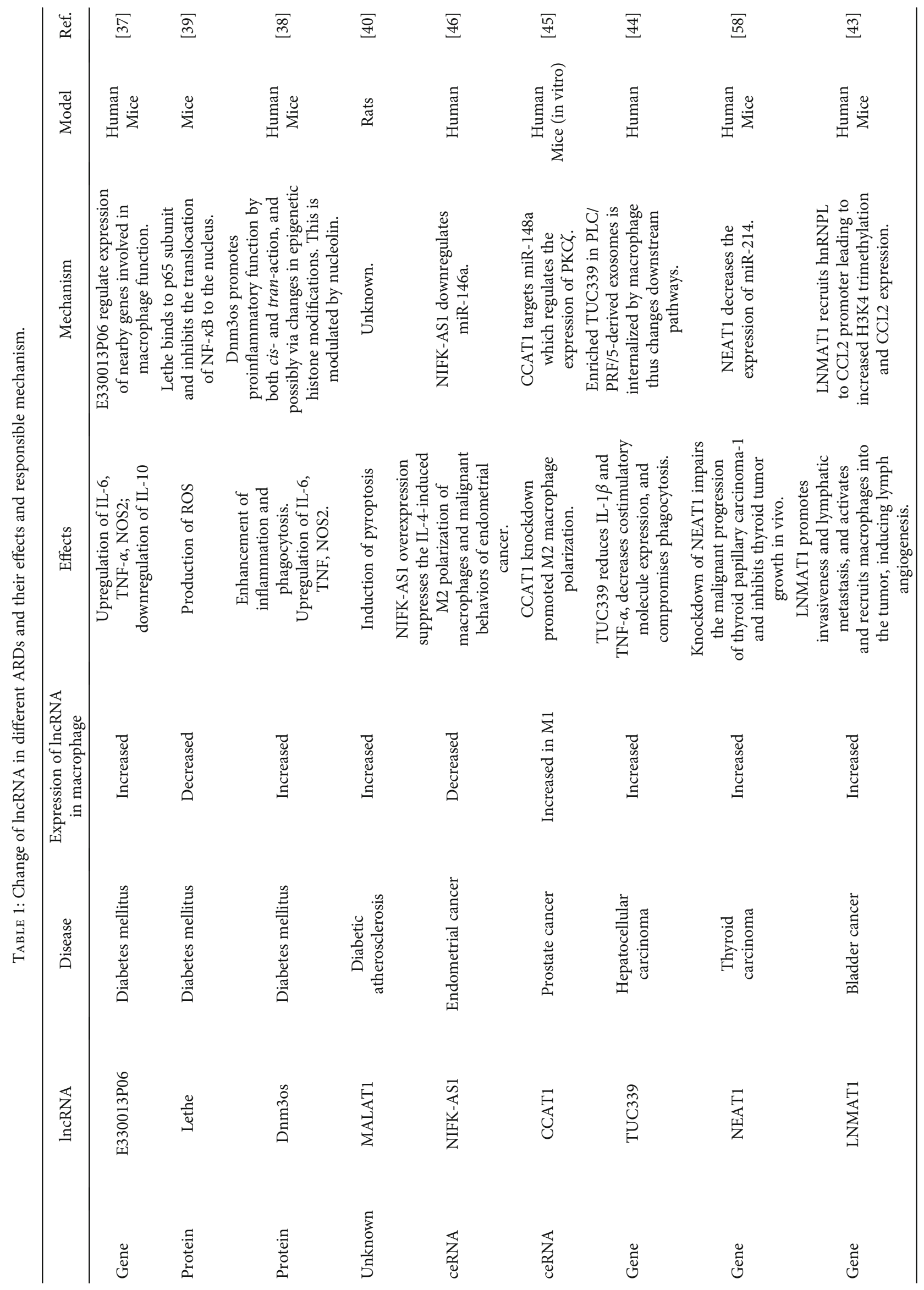




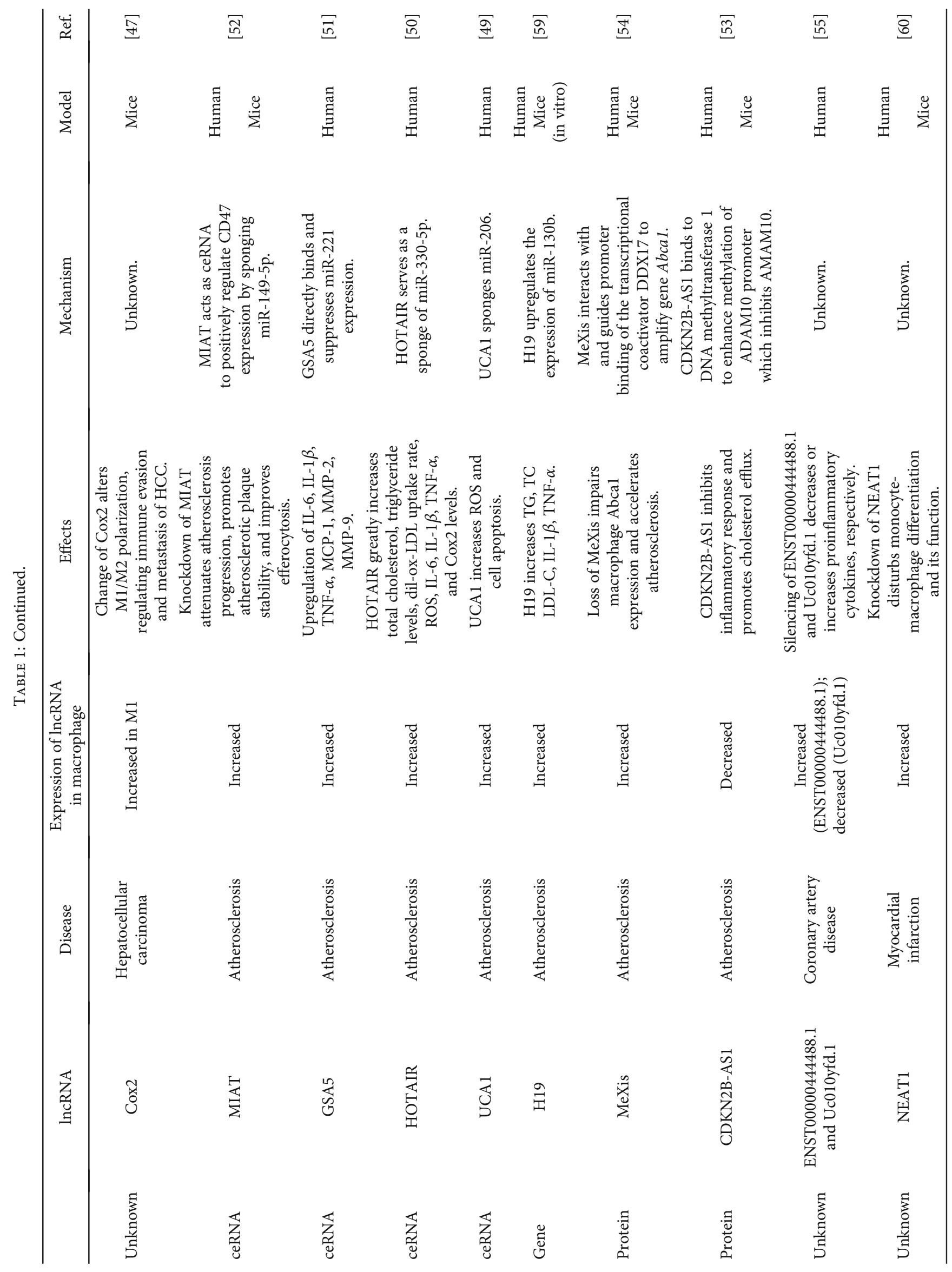




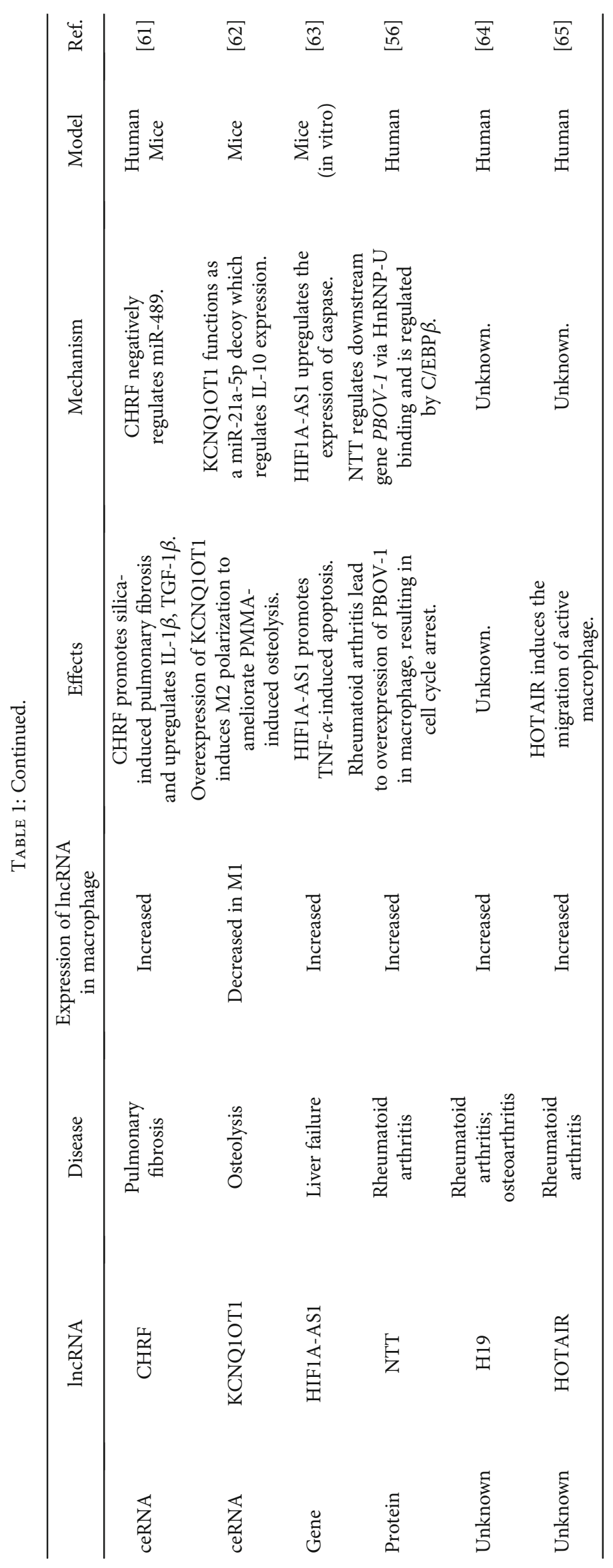


rheumatoid arthritis reported that NNT regulates downstream gene PBOV-1 via HnRNP-U binding and ultimately alters cellular senescence, i.e., cell cycle arrest [56]. This is a direct testimony to the potential of lncRNA to regulate cell lifespans.

Macrophage polarization is a fundamental phenomenon. Through the mutual transformation of macrophages M1 and M2, macrophages exert opposite functions and participate in different physiological phenomena. M1 polarization is proinflammatory and is associated with inflammaging, while M2 polarization is anti-inflammatory [57]. Increasing evidence indicates that M1 macrophages are at a higher risk of aging-related stress and display senescence phenotypes. lncRNA initiates senescence and ARD pathogenesis by altering macrophage polarization.

\section{Three Targets of lncRNA to Regulate Macrophage Senescence}

lncRNA alters cellular senescence by mediating gene expression or protein function or functioning as ceRNA. Genes regulated by $\operatorname{lncRNA}$ during aging are primarily those involved in the p53 pathway [66]. Furthermore, the expression of cellular senescence-related proteins including $\mathrm{p} 21, \mathrm{p} 27$, and $\mathrm{p} 16^{\text {Ink4a }}$ is influenced $[67,68]$. IncRNA generally functions in cis- or trans-action where the former regulates various proximal genes, while the latter regulates distal genes. However, the detailed mechanism underlying lncRNA-gene interactions remains unclear; however, studies have reported certain advancements. lncRNA H19 promotes antiaging effects through miR-675. The latter downregulates p53 and p21 by targeting the $3^{\prime}$ UTR of USP10. However, it remains to be elucidated whether there is any mediator in this interplay, along with the mechanism underlying $\mathrm{H} 19$ and miR-675 interactions [66]. Furthermore, lncRNA GUARDIN is indispensable for genomic stability by preventing chromosome end-to-end fusion through maintenance of the expression of telomeric repeat-binding factor 2 via sequestering of miR-23a. Moreover, GURADIN downregulation potentially triggers apoptosis and senescence [69]. Certain lncRNAs are involved in genomic stability. $\mathrm{R}$ loop formation and cellular senescence have reportedly occurred after alteration of the expression of certain lncRNAs [70]. Moreover, lncRNA CAIF potentially displays transcription factor-like functions trough blocking of p53-mediated myocardin transcription [71].

Along with genes, lncRNA mediates cellular senescence by functioning as ceRNA or by directly targeting proteins. CeRNA competes with microRNAs and regulates their effects on other genes, RNAs, and proteins. IncRNA regulates cellular senescence primarily in this manner. By sequestering various microRNAs, lncRNA prevents them from binding to targets, thus impairing their function [72]. Except for the regulation of gene expression, sponging potentially results in a direct functional change in proteins by attenuating key protein degradation triggered by microRNAs or relieving functional restraints of microRNAs on certain proteins $[73,74]$.
In a more direct and albeit less frequent manner, proteins become the primary target of lncRNA binding, thus altering their conformation and epigenetic modifications and initiate alterations in their downstream pathways. IncRNA potentially binds to transcription factors or RNA-binding proteins and forms polycomb repressive complexes to regulate downstream genes $[75,76]$.

\section{Mechanisms of lncRNA-Induced Macrophage Inflammaging in ARDs}

Through three targets, lncRNA manipulates complex biological behaviors. As shown in Table 1, secretome changes induced by lncRNA include SASP expression, i.e., upregulation of matrix metalloproteinase, IL- $1 \beta$, IL-6, and TNF- $\alpha$, and downregulation of IL-4 and IL-10, which contributes to inflammaging and is one of the hallmarks of macrophage senescence [77]. Another notable phenotype is oxidative stress in macrophages. ROS induction is not a rare event in these ARDs. The oxidative stress signal is in a network comprising mitochondria, the endoplasmic reticulum, and numerous mutually regulated signaling pathways. Oxidative stress and autophagy defects are probably the most prominent mutually regulated phenomena. Autophagy inhibition triggered by the induction of oxidative stress [78] causes the accumulation of damaged mitochondria and aggregative proteins and rodex homeostasis disequilibrium, leading to impaired protein-folding capacity, unstable lysosomes, and ROS [32, 79]. This cyclic pathway results in the aginginducing inflammaging and promotes the expression of SASPs. On combining existing evidence with this theory, lncRNA triggers either ROS, SASPs, or both, thus presenting a self-amplification system targeting genes, microRNA, or proteins to induce inflammaging, which accelerates cellular senescence and eventually leads to the exacerbation of ARDs. This deteriorative cycle occurs not only in disease models but also in the simple aging process [32], highlighting the viewpoint that inflammaging is a ubiquitous phenomenon that boosts senescence in aging or the proaging status and is a promising therapeutic target of ARDs.

DAMPs and PAMPs induce cellular senescence primarily through the p53 signaling pathway, AIM2 signaling pathway, and the NF- $\kappa$ B signaling pathway $[24,25]$. Upon activation, normal cellular homeostasis is prone to disruption in the presence of downstream gene products, wherein the disruption of the balance between production and clearance leads to increased SASP expression and increased inflammasomes. Excessive proinflammatory factors accelerate aging and increase the risk of ARDs. More importantly, SASPs include two vicious cycles, thus forming the cascade (Figure 2): (1) the SASP is an important pathogenic factor and attribute of inflammaging. Therein, IL-1, IL-6, IL-8, MMP-3, and other particular factors can trigger inflammaging, while cells in this proinflammatory condition express SASPs via activation of Rcor2 and the NF- $\kappa$ B signaling pathway [80], resulting in the first deteriorative cycle. (2) Macrophage senescence is inevitably accompanied by a functional decline in organelles. Increasing evidence indicates that autophagy is reduced when cells enter the late stage of senescence [81, 82]. Being 


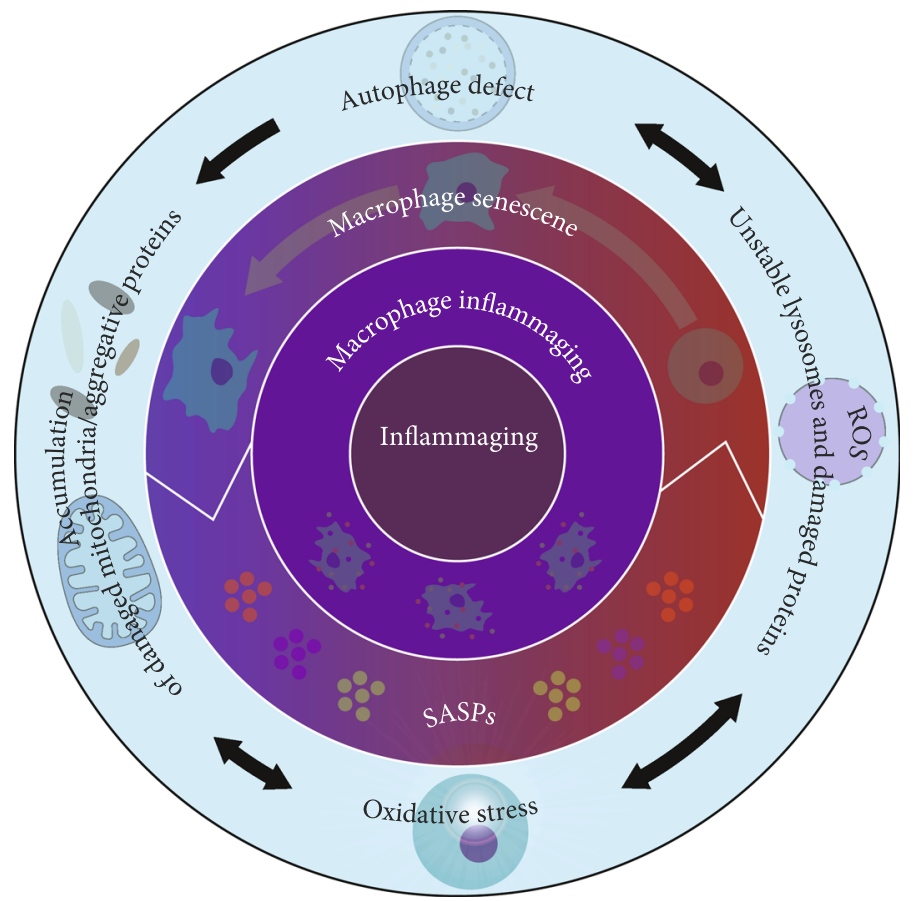

Figure 2: Schematic of the two vicious cycles.

at one complex regulatory network comprising numerous other organelles, autophagy defects consequently trigger inflammaging, forming the second deteriorative cycle.

These data undoubtedly depict a mutual association between them and insinuate the significant promoting effects of inflammaging on macrophage senescence. Recent studies on NLRP3 inflammasomes had proved this cascade model [83]. NLRP3 is assembled upon the detection of PAMPs or DAMPs. Thereafter, it activates the corresponding signaling pathway, usually NF- $\kappa \mathrm{B}$, inducing the expression of SASPs and pyroptotic cell death [84]. IncRNA regulates the activation and expression of NLRP3 by sequestering microRNA or inhibiting NF- $\kappa$ B phosphorylation in uric acid nephropathy or in the inflammatory response $[85,86]$. Indeed, some studies have reported that inflammaging can cause certain diseases [87], although studies in this field are relatively few. Thus, inflammaging may be a potential target in countering ARDs and delaying aging. Another intriguing aspect is the regulation of the intensity of inflammaging, probably through effects on lncRNAs, such that organisms safely survive the aging process and do not result in disorders [5].

\section{Conclusion}

This review discusses a delicate complement of inflammaging, which emphasizes on the disruption of the cellular onset of systemic inflammatory homeostasis. Increasing evidence indicates that $\operatorname{lncRNA}$ regulates inflammation. Alterations in the expression levels of various lncRNAs are associated with a proinflammatory phenotype in various ARDs. By directly binding to proteins or genes or indirectly serving as ceRNA, lncRNA regulates anti-inflammatory and proinflammatory processes (Figure 3). Upon disruption of macrophage homeostasis, SASPs, ROS, and other compo- nents would form a self-amplifiable aging-promoting environment to accelerate macrophage senescence and paracrine senescence and worsen ARDs. Defective autophagy, deleterious oxidative stress, and DNA damage SASP expression are the primary pathogenic mechanisms of inflammaging. Their roles are mutual, and it is largely unclear how they trigger inflammaging. Autophagy defects reduce ROS scavenging; ROS accumulation damages mitochondria, resulting in excessive deleterious oxidative stress. Inflammasomes assemble upon the recognition of DAMPs and activate downstream proinflammatory signals, inducing the expression of SASPs, including interleukin and matrix metalloproteinase secretion.

Owing to the marked inflammatory plasticity of macrophage, aberrant activation or functional alterations potentially induce ARDs. The incidence of diabetes mellitus, cancer, and atherosclerosis continues to increase. These ARDs and their complications impose a huge burden on global public health. Although numerous studies have suggested and some have proved the association between macrophage inflammaging and ARDs, the intermediate details warrant further study. Current studies are attempting to harness macrophage inflammaging in ARDs. Future researches require deeper and broader work. Theories put forward in this review link lncRNA to ARDs and summarize in detail the molecular mechanism of this linkage. They also shed light on the relationship of cellular inflammaging and macroscopic senescent phenotypes. These theories would be helpful guidance in finding novel targets for ARDs and other inflammaging-related disorders. Given that new functions of lncRNA are constantly being discovered, they shall always be considered in the pathogenic mechanism of ARDs and more lncRNA shall be found in this process. Furthermore, pharmacotherapeutic intervention of macrophage senescence by 


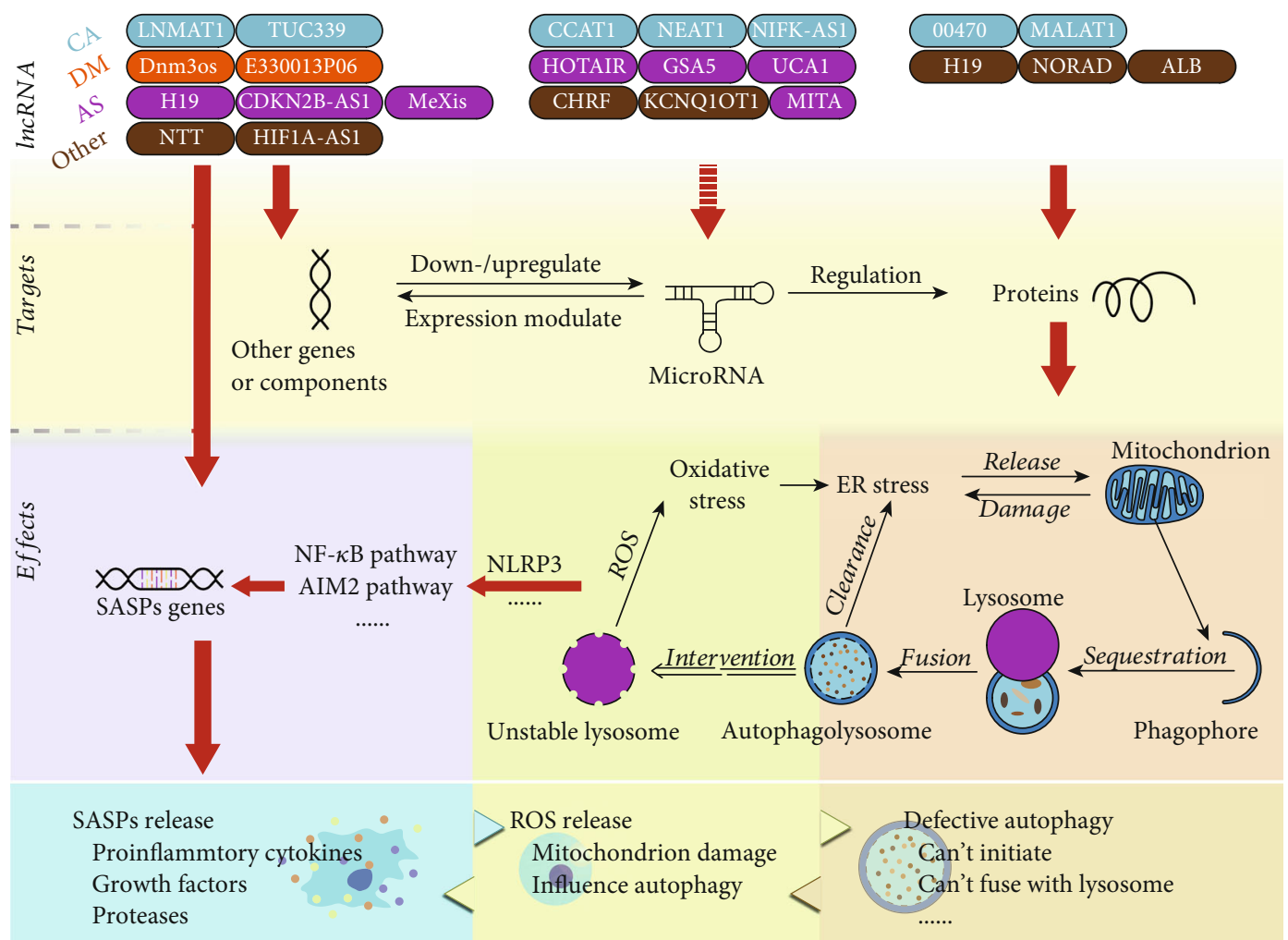

FIGURE 3: Schematic of the mechanism of how lncRNA triggers inflammaging and cellular senescence. CA: cancer; DM: diabetes mellitus; AS: atherosclerosis.

senolytic compounds, like metformin, polyphenols, aspirin, and epigallocatechin gallate [88], may be a novel method to prevent or treat ARDs. However, considering the diverse functions of senescent macrophages under different conditions, it remains to be determined whether this intervention is beneficial, probably by utilizing clodronate liposomes or INK-ATTAC $[89,90]$ to eliminate senescent macrophages.

In conclusion, the effect of IncRNA on macrophage senescence warrants further investigation, with numerous gray areas to be investigated. Targeting lncRNA during inflammaging would be an intriguing and promising approach to understand inflammaging and would help identify methods to treat ARDs.

\section{Conflicts of Interest}

The authors declare that they have no potential conflicts of interest relevant to this study.

\section{Acknowledgments}

This work was supported by the National Natural Science Foundation of China (81870779), the International Scientific Cooperation and Exchanges Project of Sichuan Province (2017HH0078), and the International Cooperation Project of Chengdu Municipal Science and Technology Bureau (2015-GH02-00035-HZ).

\section{References}

[1] F. Olivieri, F. Prattichizzo, J. Grillari, and C. R. Balistreri, "Cellular senescence and inflammaging in age-related diseases," Mediators of Inflammation, vol. 2018, Article ID 9076485, 6 pages, 2018.

[2] T. Eggert, K. Wolter, J. Ji et al., "Distinct functions of senescence-associated immune responses in liver tumor surveillance and tumor progression," Cancer Cell, vol. 30, no. 4, pp. 533-547, 2016.

[3] J. I. Jun and L. F. Lau, "The matricellular protein CCN1 induces fibroblast senescence and restricts fibrosis in cutaneous wound healing," Nature Cell Biology, vol. 12, no. 7, pp. 676-685, 2010.

[4] D. Munoz-Espin, M. Canamero, A. Maraver et al., "Programmed cell senescence during mammalian embryonic development," Cell, vol. 155, no. 5, pp. 1104-1118, 2013.

[5] K. S. Szicvel, K. Declerck, M. Vidaković, and W. Vanden Berghe, "From inflammaging to healthy aging by dietary lifestyle choices: is epigenetics the key to personalized nutrition?," Clinical Epigenetics, vol. 7, no. 1, p. 33, 2015.

[6] T. Fulop, J. M. Witkowski, F. Olivieri, and A. Larbi, "The integration of inflammaging in age-related diseases," Seminars in Immunology, vol. 40, pp. 17-35, 2018.

[7] R. Büttner, A. Schulz, M. Reuter et al., "Inflammaging impairs peripheral nerve maintenance and regeneration," Aging Cell, vol. 17, no. 6, article e12833, 2018.

[8] N. Frey, S. Venturelli, L. Zender, and M. Bitzer, "Cellular senescence in gastrointestinal diseases: from pathogenesis to therapeutics," Nature Reviews Gastroenterology \& Hepatology, vol. 15 , no. 2, pp. 81-95, 2018. 
[9] S. He and N. E. Sharpless, "Senescence in health and disease," Cell, vol. 169, no. 6, pp. 1000-1011, 2017.

[10] F. Prattichizzo, V. De Nigris, E. Mancuso et al., "Short-term sustained hyperglycaemia fosters an archetypal senescenceassociated secretory phenotype in endothelial cells and macrophages," Redox Biology, vol. 15, pp. 170-181, 2018.

[11] M. M. Kaneda, P. Cappello, A. V. Nguyen et al., "Macrophage PI3K $\gamma$ drives pancreatic ductal adenocarcinoma progression," Cancer Discovery, vol. 6, no. 8, pp. 870-885, 2016.

[12] T. T. P. Seijkens, K. Poels, S. Meiler et al., "Deficiency of the T cell regulator Casitas B-cell lymphoma-B aggravates atherosclerosis by inducing CD8+ $\mathrm{T}$ cell-mediated macrophage death," European Heart Journal, vol. 40, no. 4, pp. 372-382, 2019.

[13] Y. Zhou, X. Yu, H. Chen et al., "Leptin deficiency shifts mast cells toward anti-inflammatory actions and protects mice from obesity and diabetes by polarizing M2 macrophages," Cell Metabolism, vol. 22, no. 6, pp. 1045-1058, 2015.

[14] B. R. Nelson, C. A. Makarewich, D. M. Anderson et al., "A peptide encoded by a transcript annotated as long noncoding RNA enhances SERCA activity in muscle," Science, vol. 351, no. 6270, pp. 271-275, 2016.

[15] J. P. de Magalhaes and J. F. Passos, "Stress, cell senescence and organismal ageing," Mechanisms of Ageing and Development, vol. 170, pp. 2-9, 2018.

[16] G. P. Dimri, X. Lee, G. Basile et al., “A biomarker that identifies senescent human cells in culture and in aging skin in vivo," Proceedings of the National Academy of Sciences of the United States of America, vol. 92, no. 20, pp. 93639367, 1995.

[17] M. Narita, S. Nunez, E. Heard et al., "Rb-mediated heterochromatin formation and silencing of E2F target genes during cellular senescence," Cell, vol. 113, no. 6, pp. 703-716, 2003.

[18] A. Takahashi, N. Ohtani, K. Yamakoshi et al., "Mitogenic signalling and the $\mathrm{p} 16^{\mathrm{INK4a}}-\mathrm{Rb}$ pathway cooperate to enforce irreversible cellular senescence," Nature Cell Biology, vol. 8, no. 11, pp. 1291-1297, 2006.

[19] S. Jaiswal, M. P. Chao, R. Majeti, and I. L. Weissman, "Macrophages as mediators of tumor immunosurveillance," Trends in Immunology, vol. 31, no. 6, pp. 212-219, 2010.

[20] C. L. Lucas, H. S. Kuehn, F. Zhao et al., "Dominant-activating germline mutations in the gene encoding the $\mathrm{PI}(3) \mathrm{K}$ catalytic subunit $\mathrm{p} 110 \delta$ result in T cell senescence and human immunodeficiency," Nature Immunology, vol. 15, no. 1, pp. 88-97, 2014.

[21] U. Herbig, M. Ferreira, L. Condel, D. Carey, and J. M. Sedivy, "Cellular senescence in aging primates," Science, vol. 311, no. 5765, p. 1257, 2006.

[22] Y. Zhao, A. Tyshkovskiy, D. Munoz-Espin et al., "Naked mole rats can undergo developmental, oncogene-induced and DNA damage-induced cellular senescence," Proceedings of the National Academy of Sciences of the United States of America, vol. 115, no. 8, pp. 1801-1806, 2018.

[23] J. B. Baell, D. J. Leaver, S. J. Hermans et al., "Inhibitors of histone acetyltransferases KAT6A/B induce senescence and arrest tumour growth," Nature, vol. 560, no. 7717, pp. 253257, 2018.

[24] L. Gong, H. Gong, X. Pan et al., "p53 isoform $\Delta 113$ p53/ $\Delta 133$ p53 promotes DNA double-strand break repair to protect cell from death and senescence in response to DNA damage," Cell Research, vol. 25, no. 3, pp. 351-369, 2015.
[25] A. W. Shao, H. Sun, Y. Geng et al., "Bclaf1 is an important $\mathrm{NF}-\kappa \mathrm{B}$ signaling transducer and $\mathrm{C} / \mathrm{EBP} \beta$ regulator in DNA damage-induced senescence," Cell Death and Differentiation, vol. 23, no. 5, pp. 865-875, 2016.

[26] A. R. Bonfigli, L. Spazzafumo, F. Prattichizzo et al., "Leukocyte telomere length and mortality risk in patients with type 2 diabetes," Oncotarget, vol. 7, no. 32, pp. 50835-50844, 2016.

[27] V. Kordinas, A. Ioannidis, and S. Chatzipanagiotou, "The telomere/telomerase system in chronic inflammatory diseases. Cause or effect?," Genes, vol. 7, no. 9, p. 60, 2016.

[28] J. C. Acosta, A. Banito, T. Wuestefeld et al., "A complex secretory program orchestrated by the inflammasome controls paracrine senescence," Nature Cell Biology, vol. 15, no. 8, pp. 978-990, 2013.

[29] B. M. Hall, V. Balan, A. S. Gleiberman et al., "Aging of mice is associated with p16(Ink4a)- and $\beta$-galactosidasepositive macrophage accumulation that can be induced in young mice by senescent cells," Aging, vol. 8, no. 7, pp. 1294-1315, 2016.

[30] F. Prattichizzo, M. Bonafe, F. Olivieri, and C. Franceschi, "Senescence associated macrophages and "macroph-aging": are they pieces of the same puzzle?," Aging, vol. 8, no. 12, pp. 3159-3160, 2016.

[31] A. A. van Beek, J. Van den Bossche, P. G. Mastroberardino, M. P. J. de Winther, and P. J. M. Leenen, "Metabolic alterations in aging macrophages: ingredients for inflammaging?" Trends in Immunology, vol. 40, no. 2, pp. 113-127, 2019.

[32] A. Salminen, K. Kaarniranta, and A. Kauppinen, "Inflammaging: disturbed interplay between autophagy and inflammasomes," Aging, vol. 4, no. 3, pp. 166-175, 2012.

[33] H. Yao and I. Rahman, "Perspectives on translational and therapeutic aspects of SIRT1 in inflammaging and senescence," Biochemical Pharmacology, vol. 84, no. 10, pp. 13321339, 2012.

[34] M. Anderson Douglas, M. Anderson Kelly, C.-L. Chang et al., "A micropeptide encoded by a putative long noncoding RNA regulates muscle performance," Cell, vol. 160, no. 4, pp. 595606, 2015.

[35] H. Geng, H. F. Bu, F. Liu et al., "In inflamed intestinal tissues and epithelial cells, interleukin 22 signaling increases expression of H19 long noncoding RNA, which promotes mucosal regeneration," Gastroenterology, vol. 155, no. 1, pp. 144-155, 2018.

[36] B. Liu, B. Ye, L. Yang et al., "Long noncoding RNA _lncKdm2b_is required for ILC3 maintenance by initiation of Zfp292 expression," Nature Immunology, vol. 18, no. 5, pp. 499-508, 2017.

[37] M. A. Reddy, Z. Chen, J. T. Park et al., "Regulation of inflammatory phenotype in macrophages by a diabetes-induced long noncoding RNA," Diabetes, vol. 63, no. 12, pp. 4249-4261, 2014.

[38] S. Das, M. A. Reddy, P. Senapati et al., "Diabetes mellitusinduced long noncoding RNA Dnm3os regulates macrophage functions and inflammation via nuclear mechanisms," Arteriosclerosis, Thrombosis, and Vascular Biology, vol. 38, no. 8, pp. 1806-1820, 2018.

[39] C. Zgheib, M. M. Hodges, J. Hu, K. W. Liechty, and J. Xu, "Long non-coding RNA Lethe regulates hyperglycemiainduced reactive oxygen species production in macrophages," PLoS One, vol. 12, no. 5, article e0177453, 2017. 
[40] Y. Han, H. Qiu, X. Pei, Y. Fan, H. Tian, and J. Geng, "Low-dose sinapic acid abates the pyroptosis of macrophages by downregulation of IncRNA-MALAT1 in rats with diabetic atherosclerosis," Journal of Cardiovascular Pharmacology, vol. 71, no. 2, pp. 104-112, 2018.

[41] Y. H. Lin, M. H. Wu, C. T. Yeh, and K. H. Lin, "Long non-coding RNAs as mediators of tumor microenvironment and liver cancer cell communication," International Journal of Molecular Sciences, vol. 19, no. 12, p. 3742, 2018.

[42] S. L. Zhou, Z. J. Zhou, Z. Q. Hu et al., "Tumor-associated neutrophils recruit macrophages and T-regulatory cells to promote progression of hepatocellular carcinoma and resistance to sorafenib," Gastroenterology, vol. 150, no. 7, pp. 16461658.e17, 2016, e1617.

[43] C. Chen, W. He, J. Huang et al., "LNMAT1 promotes lymphatic metastasis of bladder cancer via CCL2 dependent macrophage recruitment," Nature Communications, vol. 9, no. 1, p. $3826,2018$.

[44] X. Li, Y. Lei, M. Wu, and N. Li, "Regulation of macrophage activation and polarization by HCC-derived exosomal lncRNA TUC339," International Journal of Molecular Sciences, vol. 19, no. 10, p. 2958, 2018.

[45] J. Liu, D. Ding, Z. Jiang, T. Du, J. Liu, and Z. Kong, "Long noncoding RNA CCAT1/miR-148a/PKC $\zeta$ prevents cell migration of prostate cancer by altering macrophage polarization," The Prostate, vol. 79, no. 1, pp. 105-112, 2019.

[46] Y. X. Zhou, W. Zhao, L. W. Mao et al., "Long non-coding RNA NIFK-AS1 inhibits M2 polarization of macrophages in endometrial cancer through targeting miR-146a," The International Journal of Biochemistry \& Cell Biology, vol. 104, pp. 25-33, 2018.

[47] Y. Ye, Y. Xu, Y. Lai et al., "Long non-coding RNA cox-2 prevents immune evasion and metastasis of hepatocellular carcinoma by altering M1/M2 macrophage polarization," Journal of Cellular Biochemistry, vol. 119, no. 3, pp. 29512963, 2018.

[48] B. Aryal and Y. Suarez, "Non-coding RNA regulation of endothelial and macrophage functions during atherosclerosis," Vascular Pharmacology, vol. 114, pp. 64-75, 2019.

[49] X. Hu, R. Ma, W. Fu, C. Zhang, and X. Du, "LncRNA UCA1 sponges miR-206 to exacerbate oxidative stress and apoptosis induced by ox-LDL in human macrophages," Journal of Cellular Physiology, vol. 234, no. 8, pp. 14154-14160, 2019.

[50] J. Liu, G. Q. Huang, and Z. P. Ke, "Silence of long intergenic noncoding RNA HOTAIR ameliorates oxidative stress and inflammation response in ox-LDL-treated human macrophages by upregulating miR-330-5p," Journal of Cellular Physiology, vol. 234, no. 4, pp. 5134-5142, 2019.

[51] J. Ye, C. Wang, D. Wang, and H. Yuan, "LncRBA GSA5, upregulated by ox-LDL, aggravates inflammatory response and MMP expression in THP-1 macrophages by acting like a sponge for miR-221," Experimental Cell Research, vol. 369, no. 2, pp. 348-355, 2018.

[52] Z. M. Ye, S. Yang, Y. P. Xia et al., "LncRNA MIAT sponges miR-149-5p to inhibit efferocytosis in advanced atherosclerosis through CD47 upregulation," Cell Death \& Disease, vol. 10, no. 2, p. 138, 2019.

[53] H. Li, S. Han, Q. Sun et al., "Long non-coding RNA CDKN2BAS1 reduces inflammatory response and promotes cholesterol efflux in atherosclerosis by inhibiting ADAM10 expression," Aging, vol. 11, no. 6, pp. 1695-1715, 2019.
[54] T. Sallam, M. Jones, B. J. Thomas et al., "Transcriptional regulation of macrophage cholesterol efflux and atherogenesis by a long noncoding RNA," Nature Medicine, vol. 24, no. 3, pp. 304-312, 2018.

[55] L. Li, L. Wang, H. Li et al., "Characterization of LncRNA expression profile and identification of novel LncRNA biomarkers to diagnose coronary artery disease," Atherosclerosis, vol. 275, pp. 359-367, 2018.

[56] C. A. Yang, J. P. Li, J. C. Yen et al., "IncRNA NTT/PBOV1 axis promotes monocyte differentiation and is elevated in rheumatoid arthritis," International journal of molecular sciences, vol. 19, no. 9, p. 2806, 2018.

[57] Z. Wang and Y. Zheng, "IncRNAs regulate innate immune responses and their roles in macrophage polarization," Mediators of inflammation, vol. 2018, Article ID 8050956, 8 pages, 2018.

[58] J. H. Li, S. Q. Zhang, X. G. Qiu, S. J. Zhang, S. H. Zheng, and D. H. Zhang, "Long non-coding RNA NEAT1 promotes malignant progression of thyroid carcinoma by regulating miRNA-214," International Journal of Oncology, vol. 50, no. 2, pp. 708-716, 2017.

[59] Y. Han, J. Ma, J. Wang, and L. Wang, "Silencing of H19 inhibits the adipogenesis and inflammation response in oxLDL-treated Raw264.7 cells by up-regulating miR-130b," Molecular Immunology, vol. 93, pp. 107-114, 2018.

[60] M. Gast, B. Rauch, A. Haghikia et al., "Long noncoding RNA NEAT1 modulates immune cell functions and is suppressed in early onset myocardial infarction patients," Cardiovascular Research, vol. 115, no. 13, pp. 1886-1906, 2019.

[61] Q. Wu, L. Han, W. Yan et al., "miR-489 inhibits silica-induced pulmonary fibrosis by targeting MyD88 and Smad3 and is negatively regulated by IncRNA CHRF," Scientific reports, vol. 6, no. 1, article 30921, 2016.

[62] X. Gao, J. Ge, W. Li, W. Zhou, and L. Xu, "LncRNA KCNQ1OT1 ameliorates particle-induced osteolysis through inducing macrophage polarization by inhibiting miR-21a-5p," Biological Chemistry, vol. 399, no. 4, pp. 375$386,2018$.

[63] Y. Wu, J. Ding, Q. Sun et al., "Long noncoding RNA hypoxiainducible factor 1 alpha-antisense RNA 1 promotes tumor necrosis factor- $\alpha$-induced apoptosis through caspase 3 in Kupffer cells," Medicine, vol. 97, no. 4, article e9483, 2018.

[64] B. Stuhlmuller, E. Kunisch, J. Franz et al., "Detection of oncofetal h19 RNA in rheumatoid arthritis synovial tissue," The American Journal of Pathology, vol. 163, no. 3, pp. 901-911, 2003.

[65] J. Song, D. Kim, J. Han, Y. Kim, M. Lee, and E. J. Jin, "PBMC and exosome-derived Hotair is a critical regulator and potent marker for rheumatoid arthritis," Clinical and Experimental Medicine, vol. 15, no. 1, pp. 121-126, 2015.

[66] B. Cai, W. Ma, C. Bi et al., "Long noncoding RNA H19 mediates melatonin inhibition of premature senescence of c-kit +cardiac progenitor cells by promoting miR-675," Journal of Pineal Research, vol. 61, no. 1, pp. 82-95, 2016.

[67] M. Montes, M. M. Nielsen, G. Maglieri et al., "The lncRNA MIR31HG regulates p16 ${ }^{\mathrm{INK} 4 \mathrm{~A}}$ expression to modulate senescence," Nature Communications, vol. 6, no. 1, p. 6967, 2015.

[68] B. Sang, Y. Y. Zhang, S. T. Guo et al., "Dual functions for OVAAL in initiation of RAF/MEK/ERK prosurvival signals and evasion of p27-mediated cellular senescence," Proceedings 
of the National Academy of Sciences of the United States of America, vol. 115, no. 50, pp. E11661-E11670, 2018.

[69] W. L. Hu, L. Jin, A. Xu et al., "GUARDIN is a p53-responsive long non-coding RNA that is essential for genomic stability," Nature Cell Biology, vol. 20, no. 4, pp. 492-502, 2018.

[70] T. Nojima, M. Tellier, J. Foxwell et al., "Deregulated expression of mammalian lncRNA through loss of SPT6 induces R-loop formation, replication stress, and cellular senescence," Molecular Cell, vol. 72, no. 6, pp. 970-984.e7, 2018, e977.

[71] C. Y. Liu, Y. H. Zhang, R. B. Li et al., "LncRNA CAIF inhibits autophagy and attenuates myocardial infarction by blocking p53-mediated myocardin transcription," Nature Communications, vol. 9, no. 1, p. 29, 2018.

[72] L. Yang, H. Wang, Q. Shen, L. Feng, and H. Jin, "Long noncoding RNAs involved in autophagy regulation," Cell Death \& Disease, vol. 8, no. 10, article e3073, 2017.

[73] H. Xiong, Z. Ni, J. He et al., "LncRNA HULC triggers autophagy via stabilizing Sirt1 and attenuates the chemosensitivity of HCC cells," Oncogene, vol. 36, no. 25, pp. 3528-3540, 2017.

[74] H. YiRen, Y. YingCong, Y. Sunwu et al., "Long noncoding RNA MALAT1 regulates autophagy associated chemoresistance via miR-23b-3p sequestration in gastric cancer," Molecular Cancer, vol. 16, no. 1, p. 174, 2017.

[75] C. Peng, W. Hu, X. Weng et al., "Over expression of long noncoding RNA PANDA promotes hepatocellular carcinoma by inhibiting senescence associated inflammatory factor IL8," Scientific Reports, vol. 7, no. 1, p. 4186, 2017.

[76] A. Roth, K. Boulay, M. Gross et al., "Targeting LINC00673 expression triggers cellular senescence in lung cancer," $R N A$ Biology, vol. 15, no. 12, pp. 1499-1511, 2018.

[77] I. M. Rea, D. S. Gibson, V. McGilligan, S. E. McNerlan, H. D. Alexander, and O. A. Ross, "Age and age-related diseases: role of inflammation triggers and cytokines," Frontiers in Immunology, vol. 9, p. 586, 2018.

[78] Q. Wang, W. Guo, B. Hao et al., "Mechanistic study of TRPM2-Ca(2+)-CAMK2-BECN1 signaling in oxidative stress-induced autophagy inhibition," Autophagy, vol. 12, no. 8, pp. 1340-1354, 2016.

[79] J. R. Cubillos-Ruiz, S. E. Bettigole, and L. H. Glimcher, "Tumorigenic and immunosuppressive effects of endoplasmic reticulum stress in cancer," Cell, vol. 168, no. 4, pp. 692-706, 2017.

[80] M. J. Alvarez-López, P. Molina-Martínez, M. Castro-Freire et al., "Rcor2 underexpression in senescent mice: a target for inflammaging?," Journal of Neuroinflammation, vol. 11, no. 1, p. 126, 2014.

[81] L. Garcia-Prat, M. Martinez-Vicente, E. Perdiguero et al., "Autophagy maintains stemness by preventing senescence," Nature, vol. 529, no. 7584, pp. 37-42, 2016.

[82] H. Tai, Z. Wang, H. Gong et al., “Autophagy impairment with lysosomal and mitochondrial dysfunction is an important characteristic of oxidative stress-induced senescence," Autophagy, vol. 13, no. 1, pp. 99-113, 2017.

[83] E. Sanchez-Lopez, Z. Zhong, A. Stubelius et al., "Choline Uptake and Metabolism Modulate Macrophage IL- $1 \beta$ and IL-18 Production," Cell metabolism, vol. 29, no. 6, pp. 13501362.e7, 2019.

[84] S. M. Man and T. D. Kanneganti, "Converging roles of caspases in inflammasome activation, cell death and innate immunity," Nature Reviews Immunology, vol. 16, no. 1, pp. 7-21, 2016.
[85] J. Hu, H. Wu, D. Wang, Z. Yang, and J. Dong, "LncRNA ANRIL promotes NLRP3 inflammasome activation in uric acid nephropathy through miR-122-5p/BRCC3 axis," Biochimie, vol. 157, pp. 102-110, 2019.

[86] M. Ma, Y. Pei, X. Wang, J. Feng, Y. Zhang, and M. Q. Gao, "LncRNA XIST mediates bovine mammary epithelial cell inflammatory response via NF- $\kappa$ B/NLRP3 inflammasome pathway," Cell Proliferation, vol. 52, no. 1, article e12525, 2019.

[87] P. Sanchis, C. Y. Ho, Y. Liu et al., "Arterial "inflammaging" drives vascular calcification in children on dialysis," Kidney International, vol. 95, no. 4, pp. 958-972, 2019.

[88] F. Gurau, S. Baldoni, F. Prattichizzo et al., "Anti-senescence compounds: A potential nutraceutical approach to healthy aging," Ageing Research Reviews, vol. 46, pp. 14-31, 2018.

[89] J. N. Farr, M. Xu, M. M. Weivoda et al., "Targeting cellular senescence prevents age-related bone loss in mice," Nature Medicine, vol. 23, no. 9, pp. 1072-1079, 2017.

[90] A. Viniegra, H. Goldberg, C. Cil et al., "Resolving macrophages counter osteolysis by anabolic actions on bone cells," Journal of Dental Research, vol. 97, no. 10, pp. 1160-1169, 2018. 


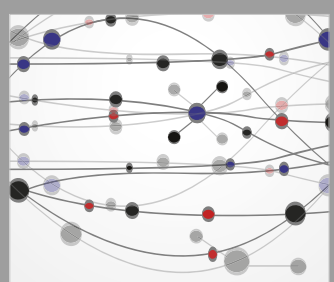

The Scientific World Journal
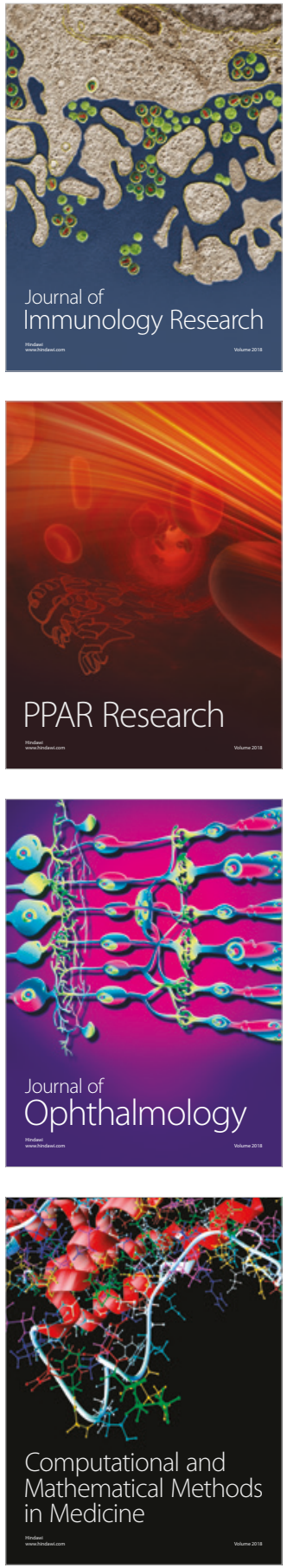

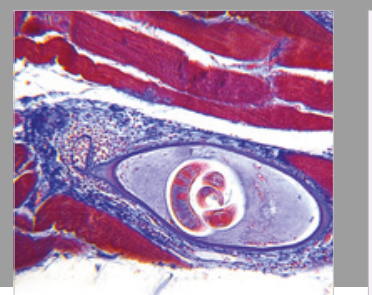

Gastroenterology Research and Practice

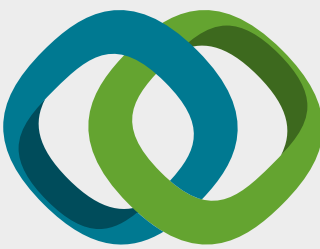

\section{Hindawi}

Submit your manuscripts at

www.hindawi.com
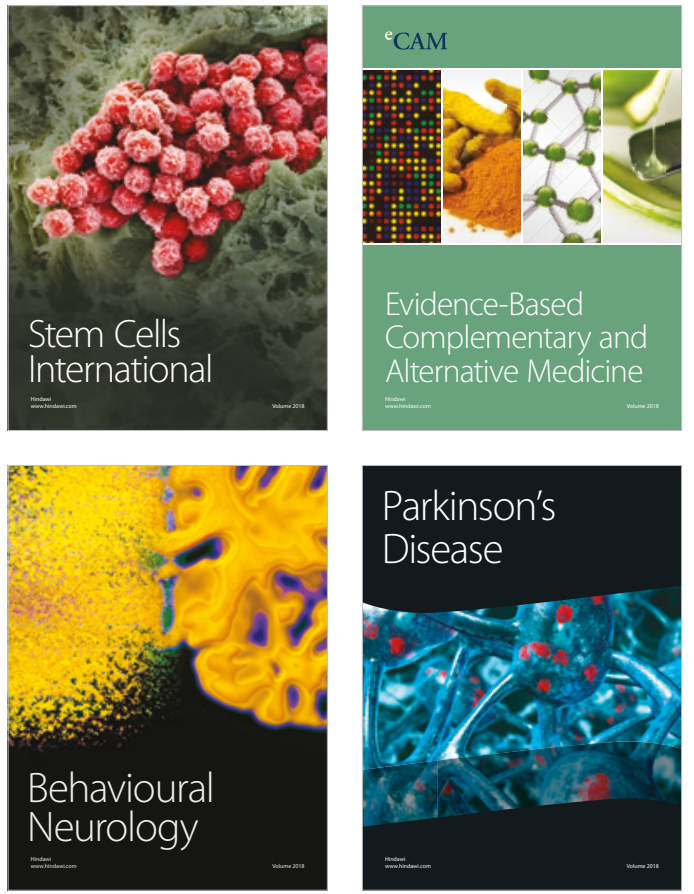

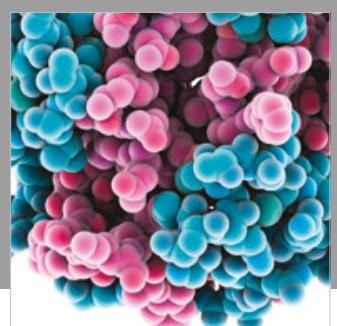

ournal of

Diabetes Research

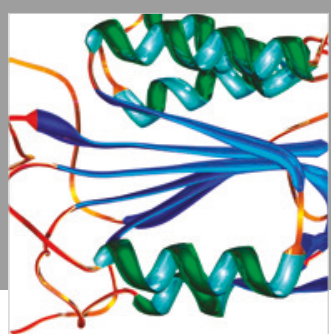

Disease Markers
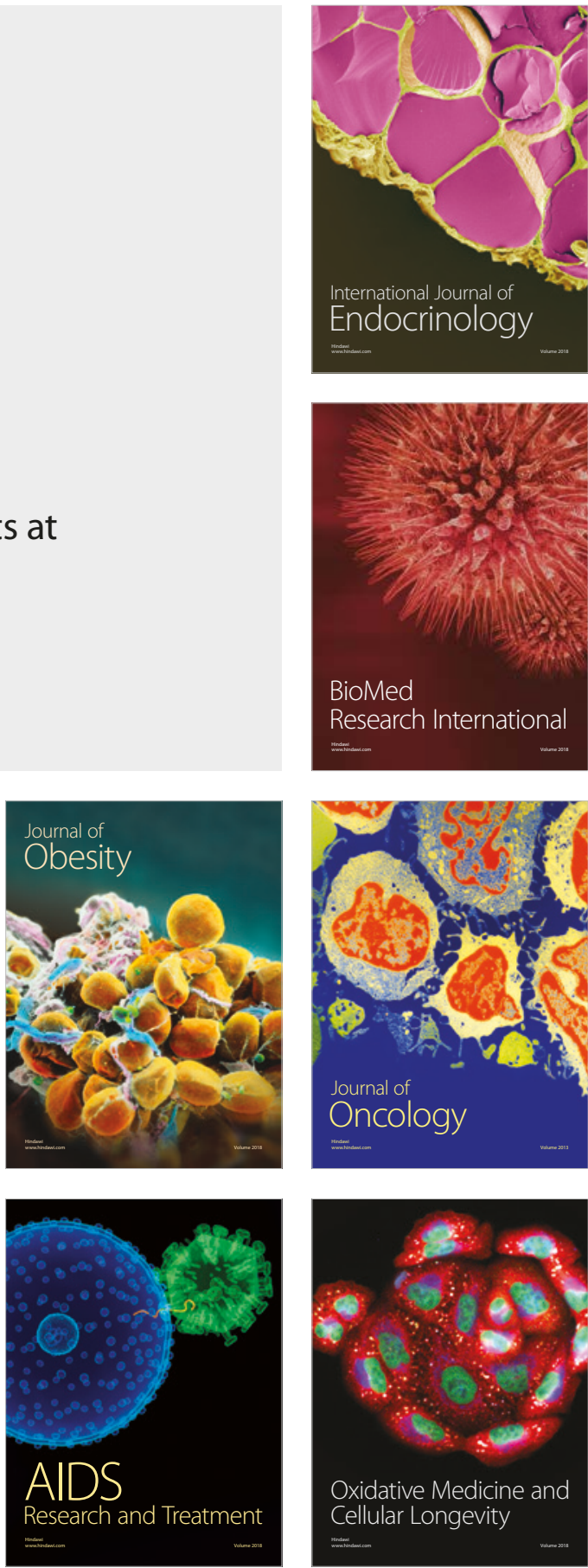